\title{
Relationship of Endoplasmic Reticulum Stress with the Etiopathogenesis of Chronic Tonsillitis and Tonsillar Hypertrophy in Pediatric Patients: A Prospective, Parallel-Group Study
}

Merih Onal ( $\nabla$ drmerihonal@gmail.com )

Selçuk University Faculty of Medicine: Selcuk Universitesi Tip Fakultesi https://orcid.org/0000-00030591-8411

\section{Nadir Kocak}

Selçuk University Faculty of Medicine: Selcuk Universitesi Tip Fakultesi

\section{Fahrettin Duymus}

Selçuk University Faculty of Medicine: Selcuk Universitesi Tip Fakultesi

\section{Mete Kaan Bozkurt}

Selçuk University Faculty of Medicine: Selcuk Universitesi Tip Fakultesi

\section{Cagdas Elsurer}

Selçuk University Faculty of Medicine: Selcuk Universitesi Tip Fakultesi

\section{Omer Erdur}

Selçuk University Faculty of Medicine: Selcuk Universitesi Tip Fakultesi

\section{Ozkan Onal}

Selçuk Üniversitesi Tip Fakültesi: Selcuk Universitesi Tip Fakultesi

\section{Research Article}

Keywords: endoplasmic reticulum stress, apoptosis, children, genetics, palatine tonsil, tonsillitis.

Posted Date: May 4th, 2021

DOl: https://doi.org/10.21203/rs.3.rs-434079/v1

License: (c) (i) This work is licensed under a Creative Commons Attribution 4.0 International License. Read Full License

Version of Record: A version of this preprint was published at Molecular Biology Reports on July 22nd, 2021. See the published version at https://doi.org/10.1007/s11033-021-06579-4. 


\section{Abstract}

Objectives: Tonsil tissue is a very important component of the human immunity system, contributing to the functioning of the cellular and humoral defence system, especially in childhood. The endoplasmic reticulum (ER) is an organelle that has a very important function in the balanced functioning of cells, in which the accumulation of a cellular protein called ER stress occurs in case of dysfunction. ER stress influences the pathogenesis of many diseases and immune system functions. We aimed to investigate the relation between the diseases of tonsil tissue and ER stress response to elucidate the mechanisms of diseases related with the immune system.

Methods: A prospective study was conducted in 46 children aged between 2 and 16 years who underwent tonsillectomy for chronic tonsillitis or tonsillar hypertrophy. Tonsil tissue was separated into two groups according to their size and evaluated in terms of ER stress markers and apoptosis markers by Real-time PCR and Western blot analysis.

Results: The $\triangle \mathrm{CT}$ levels of ER stress markers (ATF4, ATF6, CHOP, GRP78, EIF2AK3, ERN1, GRP94) were greater in children with chronic tonsillitis $(p<0.005)$. In contrast, the tonsillar hypertrophy group had greater $\triangle C T$ levels of apoptosis markers (BAX, BCL-2) according to the Real-time PCR method $(p<0.005)$. According to the Western blot analysis, the normalized levels of ATF4, ATF6, CHOP, GRP78, and ERN1 genes were found greater in the chronic tonsillitis group than the tonsillar hypertrophy group. There was no difference between the two groups in terms of normalized BCL-2 and BAX levels by Western blot analysis.

Conclusion: This is the first study in the literature investigating the effect of the ER stress pathway on the etiopathogenesis of tonsil diseases. It was concluded that the ER stress pathway plays a role in the etiopathogenesis of chronic tonsillitis. Investigating the relationship between ER stress and structures such as the tonsil tissue that make up the immune system can help create new treatment strategies.

\section{Trial Registration ClinicalTrials.gov Identifier: NCT04653376}

\section{Introduction}

Tonsil tissue, as a vital part of the Waldeyer ring, is an important component of the immune system located centrally at the entrance of respiratory and gastrointestinal systems, where pathogens, infectious agents, and allergens invading the upper respiratory tract first encounter the immune system [1]. Tonsil tissue is a secondary lymphoid organ that exerts both humoral and cellular immunity functions against various antigens with specific antibodies, $B$ and $T$ cell activities, and protects the body especially in young children [2]. Hence, in patients with tonsillitis, cellular, and humoral immune responses are stimulated together. Immunological reactions in tonsil tissue may give rise to chronic infections and hypertrophy. Chronic tonsillitis is the state of persistent inflammation associated with recurrent acute 
tonsillitis or subclinical infections. Tonsillar hypertrophy develops as a consequence of fibroid degeneration which starts in early childhood and continues until puberty and produces parenchymal hyperplasia or obstruction in crypts. Hypertrophy may also occur following bouts of local or systemic infection. However, they may also undergo atrophy with chronic disease processes [2].

Although chronic tonsillitis and tonsillar hypertrophy seem to have similar definitions and etiology, they are histopathologically distinct entities and their effect on immune cell compositions still remains to be understood. Though the cause of these two different changes in tonsil tissue is not completely clear, diet, genetics, and humoral changes may play role in etiology [3]. However, in some studies, it was reported that chronic tonsillitis or tonsillar hypertrophy result from the hypofunction of local and systemic immunity [4]. Nevertheless, there is a paucity of data on immune responses among these patients. Tonsil tissue removed surgically is an accessible source in order to examine the relationship between foreign pathogens, allergens, and the host immune systems. Tonsil tissue also provides a suitable in vivo model for investigating the inflammatory processes in lymphoid organs and infection mechanisms [5].

The Endoplasmic Reticulum (ER), which is an organelle with many important intracellular functions, is also critical for protein synthesis, folding and modification, and lipid synthesis, and calcium storage. ER synthesizes around one-third of the total proteome and plays a dominant role in the folding and maturation of proteins [6]. When the functional capacity of ER is exceeded or when ER functions become irregular, misfolded, or unfolded protein accumulation takes place in the ER lumen and this condition is termed as "ER stress" [7]. After ER stress develops in the cell, for the maintenance of vital functions of cells, a condition called [Unfolded Protein Response (UPR)], which reverses ER homeostasis, is triggered. UPR is characterized by different pathways inducing the survival or apoptosis of cells depending on the agent, intensity, and duration of ER stress, and the type of cell [6]. It was shown that the UPR response regulates intracellular metabolic, oxidative stress, and inflammation pathways [7].

The three main transmembrane proteins that occur as a result of the ER stress response and initiate the UPR signal are Activating transcription factor 6 (ATF6), protein kinase R-like ER kinase (PERK), and enzyme 1 (IRE1) that requires inositol [8]. These are normally in an inactive state. If ER stress is triggered by any pathology, they become active by separating from GRP78/binding protein (BIP), the main regulatory chaperon of UPR.

In particular, ATF6 induces the expression of transcription factors that regulate UPR elements after displacement to the nucleus and stabilizes both ER-associated protein degradation (ERAD) and ERchaperones protein folding to reduce unfolded protein accumulation [9].

The active form of PERK phosphorylates both Eukaryotic initiation factor 2 (elF2), which is involved in protein translation of ATF4 and induces protein folding factor gene expression to regulate ER homeostasis. IRE1 is the most conserved modulator of the ER stress signal pathway. However, if ER stress persists and UPR cannot provide hemostasis, apoptotic pathways are induced [10]. The first key molecule for ER stress-mediated apoptosis is the C/EBP homologous protein (CHOP), which increases 
cellular reactive oxygen species (ROS) production and $\mathrm{Ca} 2{ }^{+}$concentration. Thus, the $\mathrm{CHOP}$ protein reduces the antiapoptotic expression of both B-cell lymphoma 2 (Bcl-2) and Bcl-2-associated X protein (Bax) [11].

The direct relation between ER stress and immunity responses is clear but as the casual relation between ER stress and immunity remains undetermined, this issue still attracts attention. In addition, mechanisms coordinating UPR signaling cascades with immunity still remains to be elucidated. Further studies are required in order to define the role of UPR in relevant regulatory mechanisms, signal molecules, and immunity. It was demonstrated that ER stress contributes significantly to the pathogenesis of various diseases including cancer, neurodegenerative disorders, inflammatory and autoimmune diseases, expression of inflammatory cytokines, and induction of inflammatory responses and enhances their severity [6]. If chronic or intense ER stress is not resolved, UPR induces apoptosis [7]. Tonsil tissue inhibits immune response in cases of chronic inflammation. However, whether inhibition of immune responses occurs via ER stress and UPR has not been examined so far. ER stress response in the immune system was investigated only in intestines up to date [12].

The present study aimed to investigate the role of ER stress response in molecular pathophysiological processes of chronic tonsillitis and tonsillar hypertrophy, two different pathologic entities, which occur in tonsil tissue where differentiation and maturation of immune cells take place and to contribute to the development of new treatment strategies targeting UPR pathways.

\section{Primary outcome}

Our primary outcome was to investigate whether ER stress response and apoptosis play a role in the etiopathogenesis of chronic tonsillitis and tonsillar hypertrophy, which are tonsil tissue diseases that play an important role in the functioning of the immune system of children.

\section{Materials And Methods}

Approval was obtained from the Ethics Committee of Selcuk University Medical Faculty for the study (2018/329). Our study was supported by the Selcuk University Scientific Research Projects Coordination Unit (Project number: 19401029). After obtaining informed consent from the parents of the patients to be included in the study, we conducted our study by the ethical standards stated in the 1964 Helsinki Declaration. Human tonsil samples used in this study were obtained from tonsillectomy patients in Selcuk University, Medicine Faculty Hospital Department of Ear Nose Throat (ENT) between the dates of November 2018 and August 2019.

\section{Study population, sample collection, and protocol}

Our study was conducted on 46 patients (25 males, 21 females) aged between 2 and 16 years (mean age 6.2), who were clinically diagnosed with chronic tonsillitis and tonsillar hypertrophy by the ENT clinician. Patients with a history of immunodeficiency or systemic disease were excluded from the study. The 
tonsillectomy procedure was performed under general anesthesia using the classical dissection method. After the palatal pharyngeal archus mucosa was cut with low-heat plasma and the tonsil capsule was opened, a cryogenic plasma knife was used to remove the tonsil tissue and stop bleeding. Extracted tonsil tissue was classified as 1, 2, 3, and 4 using the Friedman staging system according to their size [13].

Chronic tonsillitis group ( $\boldsymbol{n}=\mathbf{2 3}$ ); Patients with tonsil sizes grade 1 and 2 , and tonsillectomy indications included frequently recurrent tonsillary infection, sore throat, and malodorous mouth problems were accepted as the chronic tonsillitis group.

Tonsillar hypertrophy group $(n=23)$; Patients with tonsil size grades 3 and 4 and tonsillectomy indications included obstructive symptoms such as snoring, open mouth breathing, difficulty in breathing, and swallowing problems were accepted as the tonsillar hypertrophy group.

Extracted tonsil tissue samples were sent to the medical genetics department in TRIzol tubes to investigate the ER stress and apoptosis. Two consultants (NK, FD) from the medical genetics department who blinded to the study groups and the clinical characteristics of the patients, evaluated tonsil tissue samples for ER stress and apoptosis using Real-time PCR and Western blot methods.

\section{Measurements}

Tonsillar hypertrophy and chronic tonsillitis groups were compared in terms of expression levels of genes in the ER stress pathway (ATF4, ATF6, EDEM1, CHOP, GRP78, EIF2AK3, ERN1, GRP94) and apoptosis pathway (BAX, BCL-2).

\section{Real-time PCR analysis}

Total RNA extraction from tissue samples obtained from patients was performed using TRIzol® reagent (Invitrogen, Waltham, USA) according to the protocol previously described in the literature [14]. Tissue pieces were dissected by freezing in liquid nitrogen before extraction. cDNA synthesis was performed using the Transcriptor High-Fidelity cDNA Synthesis kit (Roche, Basel, Switzerland) using oligo (dT) and random primers, following the manufacturer's instructions. Oligonucleotide primers were designed using the IDT DNA primer request tool and are manufactured by Biomers Inc. (UIm, Germany). Primer sequences are given below. All PCR reactions were performed using LightCycler ${ }^{\circledR} 480$ Instrument II (Roche, Penzberg, Germany) Real-time PCR via SYBR Green Master Mix (Bio-Rad Hercules, California, USA). Before proceeding with the qPCR process of the genes of interest, optimization processes of the primers used were carried out. Housekeeping genes such as 18S rRNA (18S ribosomal RNA), 28S rRNA (28S ribosomal RNA), ACTB ( $\beta$-actin), $\beta 2 \mathrm{M}$ ( $\beta 2$-microglobulin), GAPDH (glyceraldehyde-3-phosphate dehydrogenase) were examined for normalization. ACTB levels of these were found to be more stable, and studies continued with them. $\triangle \mathrm{CT}$ results were normalized by ACTB. Expression results were calculated by the $2 \Delta \Delta$ ct method. Primer sequences were shown in Table 1. 
Antibodies and Western blot ATF-6 (D4B8) rabbit anti-human monoclonal (\# 65880), ATF-4 (D4B8) rabbit anti-human monoclone (\# 11815), GRP78 (C50B12) rabbit anti-human monoclonal mAb (\# 3177), CHOP (D46F1) ) rabbit anti-human monoclonal antibody (\# 5554), PERK (C33E10) Rabbit antihuman mAb (\#3192), ERN1 (IRE1a) (14C10) Rabbit antihuman mAb (\#3294), Grp94 (D6X2Q) XP® Rabbit antihuman mAb (\#20292), Bax (D2E11) Rabbit antihuman mAb (\#5023), Bcl-2 (D55G8) Rabbit Antihuman mAb (\#4223) and $\beta$-Actin (13E5) rabbit anti-human monoclonal antibody (\# 4970) were obtained from cell signaling technology company (cell signaling tec., Leiden, Netherlands). Western blot analyzes were performed according to protocols previously described in the literature [15]. ACTB levels were used for normalization in Western blot analysis. For each group normalization, their own ACTB values were taken into account in this calculation. Changes in protein levels were determined after this normalization.

\section{Statistical analysis}

PCR primers were designed using IDT PrimerQuest software. Image-based data were analyzed by ImageJ software. The statistical significance was investigated using GraphPad Prism V6 software (GraphPad software Inc., La Jolla, USA). Expression results were calculated by the $2 \Delta \Delta$ ct method. CT results were normalized by ACTB. All the experiments were performed as minimum triplicate $(N \geq 3)$. The SPSS version 22.0 (IBM Statistics) was used to analyze the data of the study. First of all, the conditions of the normal distribution of the data were examined with skewness and kurtosis values (values between 0.846 and 0.924) and Q_Q plot graphics, and it was observed that the data showed a normal distribution. Based on these results, an independent group t-test was used to compare the ER stress and apoptosis gene levels of chronic tonsillitis and tonsillar hypertrophy groups. The level of significance was set to $p<0.05$.

\section{Results}

In the chronic tonsillitis group, the $\triangle \mathrm{CT}$ levels of all ER stress genes except EDEM1 were found greater than the tonsillar hypertrophy group. These levels were considered as statistically significant $(p<0.005)$. And also, there was a significant difference between groups in $\triangle \mathrm{CT}$ levels of apoptosis genes i.e., BAX, BCL-2 $(p<0.005)$. But, the apoptosis pathway $\Delta C T$ levels of the tonsillar hypertrophy group were significantly greater than the chronic tonsillitis group in contrast to ER stress $\Delta C T$ levels. Table 2, Figure 1.

And also, we wanted to verify the results that we detected with the Real-time PCR method by using the Western blot method. According to the Western blot analysis, the normalized protein levels of ATF4, ATF6, CHOP, GRP78, and ERN1 genes were found in the chronic tonsillitis group 2.63, 7.56, 2.28, 2.34, and 2.41 times greater, respectively, compared to the tonsillar hypertrophy group. These increases were considered significant. There was no significant difference in the levels of the apoptosis-associated BAX, BCL-2 genes in terms of Western blot analysis. Figure 2.

\section{Discussion}


The most common indications of tonsillectomy, which is one of the most common operations performed in children all over the world, are chronic tonsillitis and tonsillar hypertrophy. It was demonstrated that immunological parameters, genetic susceptibility, and local lymphocyte dysfunction play role in the etiology of chronic tonsillitis and tonsillar hypertrophy, which share common histological properties [16]. In this study, it was established that ER stress response and apoptosis pathway also play an important role in the pathogenesis of chronic tonsillitis and tonsillar hypertrophy.

When dysfunction occurs in the ER, all cells are programmed to respond to it. However, when the ER stress process is prolonged and intensified, the proapoptotic process becomes dominant and ER stress can cause cell apoptosis [17]. The role of ER stress, an important mechanism mediating apoptosis, has not been investigated in the etiopathogenesis of chronic tonsillitis and tonsillar hypertrophy. In a previous study in which tonsillar hypertrophy and recurrent tonsillitis were compared, it was concluded that apoptosis has a role in the pathogenesis of tonsillar hypertrophy [16].

Apoptosis is actually an indicator of proliferative activity. In other words, in cases where cellular proliferation is high such as tonsillar hypertrophy, an increase in cellular apoptosis would be expected to limit cell proliferation. According to the results of the real-time PCR of our study, the $\triangle C T$ levels of apoptosis proteins in the tonsillar hypertrophy group were found to be greater than in the chronic tonsillitis group.

On the other hand, apoptosis also occurs when there is an increase in lymphocytes secondary to recurrent infections such as chronic tonsillitis. In this case, apoptosis is thought to control lymphocyte increase and cellular hyperplasia [16]. The absence of significant difference between the two groups in terms of apoptosis genes according to Western blot analysis in contrast to Real-time PCR may be due to the occurrence of apoptosis in both pathological conditions.

Ji et al., [18] examined the effect of e-cigarette aerosols on normal human keratocytes and the role of ER stress in this process was detected via the increase in the expression of CHOP, ATF4, XBP1, and IRE1 a genes. In the present study, higher expression of GRP78, CHOP, EDEM1, ATF6, and ATF4, which are ER stress markers, was observed in chronic tonsillitis groups than in the tonsillar hypertrophy group. In a human study investigating the effect of ER stress and apoptosis on osteolysis, ER stress and apoptosis were evaluated to cause loosening and loss of the prosthesis, and the apoptosis expression genes (BCL-2 and BAX) and ER stress markers (IRE1-a, GRP78 / Bip, CHOP, cleaved caspase-4, and JNK) expression levels were found to be significantly increased compared to the control group and there was a correlation between the degree of ER stress and the clinical severity of osteolysis. Thus, it was proven that ER stress exerts an impact on the apoptotic process in osteolysis [19]. Studies on human epithelial cells have investigated the role of ER stress in the development of epithelial-to-mesenchymal transition, in multiple tissues, including the eye, lungs, liver, and kidneys, and it has been revealed that ER stress triggers the development of epithelial-to-mesenchymal transition and causes pathological conditions [20].

The role of ER stress, which is an unfolded protein response, in the development of organ fibrosis is accepted. It was determined that ER stress played role in peritoneal fibrosis and apoptosis and that 
preconditioning produced by ER stress may prevent fibrosis in patients having peritoneal fibrosis [20].

In a study evaluating the antitumorigenic effects of Arachidonic acid (AA) in HT-29 human colon cancer cells, the resulting ER stress in HT-29 cells treated with AA was demonstrated by an increase in the level of the processed form of XBP1 and phosphorylated elF2a [21]. The resultant ER stress and induced apoptosis were thought to result from the provision of more AA to membrane lipids and altered membrane properties of the ER. In another study investigating the antitumor effects of a bioactive anticancer compound allyl isothiocyanate (AITC) on human colorectal cancer HT-29 cells, it has been shown that the main mechanism is the ER stress-induced by AITC-induced cytosolic $\mathrm{Ca}^{2+}$ release and the subsequent apoptosis mechanism [22].

In another study to confirm whether ER stress contributes to Mn-induced cell death, pretreatment with the ER stress inhibitor 4-PBA has been shown to reduce Mn-induced neurotoxicity. Significant increase in the expression of GRP78, PERK, elF2 $a$ and ATF4 proteins in ER stress signaling pathways with Mn incubation showed that Mn-induced neurotoxicity occurred through the ER stress pathway. It was also shown in the same study that ER stress induces CHOP and caspase-12 mediated apoptosis [23].

The molecular mechanism of the anti-cancer activity of DWP05195, a novel transient receptor potential vanilloid 1 (TRPV1) antagonist, was investigated in human ovarian cancer cells. DWP05195 has been shown to induce apoptosis by inducing (CHOP) expression and ER stress. In the same study, with the ERstress inhibitor 4-PBA, DWP5195-induced cell death was significantly suppressed [24].

\section{Conclusion}

According to our study results, although it can be said that ER stress may play role in the pathogenesis of chronic tonsillitis, and apoptosis may play role in the pathogenesis of tonsillar hypertrophy, it can not account for the pathogenesis of this complex process on its own. Information regarding the pathogenesis of tonsil diseases is very limited. It is necessary to carry out new studies on this subject both to reveal the pathogenesis of tonsil diseases and to develop alternative treatments, especially for disorders related to the immune system.

\section{Declarations}

Acknowledgements: This work was supported by the Selcuk University Scientific Research Projects Coordination Unit under Grant number 19401029.

Conflict of interest: The authors declare that they have no conflict of interest.

Ethics approval and consent to publish: All procedures performed in studies involving human participants were in accordance with the ethical standards of the institutional and/or national research committee (Selcuk University Faculty of Medicine Clinical Researches Ethics Committee Ref No: 2018/329) and with the 1964 Helsinki declaration and its later amendments or comparable ethical standards. 
Consent to participate: Informed consent was obtained from all patients' parents.

\section{Authors' contributions}

conceiving and designing the study; or collecting the data; or analyzing and interpreting the data; $M O, N K$, $F D, C E, O E, O O$,

writing the manuscript or providing critical revisions that are important for the intellectual content; $M O$, $O O, M K B$

approving the final version of the manuscript; $M O, M K B, C E, O E$,

\section{References}

1. Ogra PL (2000) Mucosal immune response in the ear, nose and throat. Pediatr Infect Dis J 19(5 Suppl):S4-S8. doi:10.1097/00006454-200005001-00002

2. Yan Y, Song Y, Liu Y, Su J, Cui L, Wang J, Geng J, Liu X, Shi Y, Quan S, Hang A, Zuo L (2019) Short and long-term impacts of adenoidectomy with/without tonsillectomy on immune function of young children < 3 years of age A cohort study. Med (Baltim) 98(19):e15530. doi:10.1097/MD.0000000000015530

3. Alan D, Kornblut A (1991) Non neoplastic diseases of the tonsils and adenoids. In: Paparella M, Shumrick DA, Gluckman JL, Meyerhof WL (eds) Otorhinolaryngology, 3, 3rd edn. New York W. B. Sounders Company, pp 2129-2147

4. Hata M, Asakura K, Saito H, Morimoto K, Kataura A (1996) Profile of immunoglobulin production in adenoid and tonsil lymphocytes. Acta Otolaryngol Suppl 523:84-86

5. Palomares O, Rückert B, Jartti T, Kücüksezer UC, Puhakka T, Gomez E, Fahrner EB, Speiser A, Jung A, Kwok WW, Kalogjera L, Akdis M, Akdis CA (2012) Induction and maintenance of allergen-specific FOXP3 + Treg cells in human tonsils as potential first-line organs of oral tolerance. J Allergy Clin Immunol 129(2):510-520. doi:10.1016/j.jaci.2011.09.031. 520.e1-9.

6. So JS (2018) Roles of Endoplasmic Reticulum Stress in Immune Responses. Mol Cells 31(8):705716. doi:10.14348/molcells.2018.0241. 41 ).

7. González-Quiroz M, Blondel A, Sagredo A, Hetz C, Chevet E, Pedeux R (2020) When Endoplasmic Reticulum Proteostasis Meets the DNA Damage Response. Trends Cell Biol 6;S0962-8924(20)301719. doi: 10.1016/j.tcb.2020.09.002

8. Amodio G, Moltedo O, Fasano D, Zerillo L, Oliveti M, Di Pietro P, Faraonio R, Barone P, Pellecchia MT, De Rose A (2019) PERK-Mediated Unfolded Protein Response Activation and Oxidative Stress in PARK20 Fibroblasts. Front Neurosci-Switz 27:13:673. doi:10.3389/fnins.2019.00673

9. Gardner BM, Walter $P$ (2011) Unfolded proteins are Ire1-activating ligands that directly induce the unfolded protein response. Science 30(6051):1891-1894. doi:10.1126/science.1209126. 333 ) . 
10. Urra H, Dufey E, Lisbona F, Rojas-Rivera D, Hetz C (2013) When ER stress reaches a dead end. BiochimBiophys Acta 1833(12):3507-3517. doi:10.1016/j.bbamcr.2013.07.024

11. Puthalakath H, O'Reilly LA, Gunn P, Lee L, Kelly PN, Huntington ND, Hughes PD, Michalak EM, McKimm-Breschkin J, Motoyama $\mathrm{N}$ et al. ER stress triggers apoptosis by activating BH3-only protein Bim. Cell 29;129(7):1337-49. doi: 10.1016/j.cell.2007.04.027

12. Wang S, Kaufman RJ (2012) The impact of the unfolded protein response on human disease. J Cell Biol 25(7):857-867. doi:10.1083/jcb.201110131. 197 ) .

13. Friedman M, Tanyeri H, Rosa ML, Landsberg R, Vaidyanathan K, Pieri S, Caldarelli D (1999) Clinical Predictors of Obstructive Sleep Apnea. Laryngoscope 109(12):1901-1907. doi:10.1097/00005537199912000-00002

14. Rio DC, Ares MJ, Hannon GJ, Nilsen TW (2010) Purification of RNA using TRIzol (TRI reagent). Cold Spring Harb Protoc (6):pdb.prot5439. doi: 10.1101/pdb.prot5439

15. Nami B, Ghasemi-Dizgah A, Vaseghi A (2016) Overexpression of molecular chaperons GRP78 and GRP94 in CD44hi/CD24lo breast cancer stem cells. Bioimpacts 6(2):105-110. doi:10.15171/bi.2016.16

16. Onal M, Yılmaz T, Bilgic E, Müftüoğlu SF, Kuşçu O, Günaydın RO (2015) Apoptosis in chronic tonsillitis and tonsillar hypertrophy. Int J Pediatr Otorhinolaryngol 79(2):191-195. doi:10.1016/j.ijporl.2014.12.005

17. Liu X, Liu JK (2020) Tanshinone I induces cell apoptosis by reactive oxygen species-mediated endoplasmic reticulum stress and by suppressing p53/DRAM-mediated autophagy in human hepatocellular carcinoma. Artif Cells Nanomed Biotechnol 48(1):488-497. doi:10.1080/21691401.2019.1709862

18. Ji EH, Elzakra N, Chen W, Cui L, Lee ES, Sun B, Messadi D, Xia T, Zhu Y, Hu S (2019) E-cigarette aerosols induce unfolded protein response in normal human oral keratinocytes. J Cancer 19(27):6915-6924. doi:10.7150/jca.31319. 10 ) .

19. Liu G, Guo T, Zhang Y, Liu N, Chen J, Chen J, Zhang J, Zhao J (2017) Apoptotic pathways of macrophages within osteolytic interface membrane in periprosthestic osteolysis after total hip replacement. APMIS 125(6):565-578. doi:10.1111/apm.12679

20. Shin HS, Ryu ES, Oh ES, Kang DH (2015) Endoplasmic reticulum stress as a novel target to ameliorate epithelial-to-mesenchymal transition and apoptosis of human peritoneal mesothelial cells. Lab Invest 95(10):1157-1173. doi:10.1038/labinvest.2015.91

21. Bae S, Kim M-K, Kim HS, Moon Y-A (2020) Arachidonic acid induces ER stress and apoptosis in HT29 human colon cancer cells. Anim Cells Syst (Seoul). 1;24(5):260-266. doi:

10.1080/19768354.2020.1813805

22. Chiang J-H, Tsai F-J, Hsu Y-M, Yin M-C, Chiu H-Y, Yang J-S (2020) Sensitivity of allyl isothiocyanate to induce apoptosis via ER stress and the mitochondrial pathway upon ROS production in colorectal adenocarcinoma cells. Oncol Rep 44(4):1415-1424. doi:10.3892/or.2020.7700 
23. Liu C, Yan D-Y, Wang C, Ma Z, Deng Y, Liu W, Xu B (2020) Manganese activates autophagy to alleviate endoplasmic reticulum stress-induced apoptosis via PERK pathway. J Cell Mol Med 24(1):328-341. doi:10.1111/jcmm. 14732

24. Wang Y-Y, Lee K-T, Lim MC, Choi JH (2020) TRPV1 Antagonist DWP05195 Induces ER StressDependent Apoptosis through the ROS-p38-CHOP Pathway in Human Ovarian Cancer Cells. Cancers (Basel) 26;12(6):1702. doi: 10.3390/cancers 12061702

\section{Tables}

Table 1. Primers information used in the present study. 


\begin{tabular}{|c|c|}
\hline Gene & Sequence \\
\hline \multicolumn{2}{|l|}{ EDEM1 } \\
\hline Forward Primer & CGGACGAGTACGAGAAGCG \\
\hline Reverse Primer & CGTAGCCAAAGACGAACATGC \\
\hline \multicolumn{2}{|l|}{ ATF4 } \\
\hline Forward Primer & ATGACCGAAATGAGCTTCCTG \\
\hline Reverse Primer & GCTGGAGAACCCATGAGGT \\
\hline \multicolumn{2}{|l|}{ ATF6 } \\
\hline Forward Primer & TCCTCGGTCAGTGGACTCTTA \\
\hline Reverse Primer & CTTGGGCTGAATTGAAGGTTTTG \\
\hline \multicolumn{2}{|l|}{ GRP78 } \\
\hline Forward Primer & CATCACGCCGTCCTATGTCG \\
\hline Reverse Primer & CGTCAAAGACCGTGTTCTCG \\
\hline \multicolumn{2}{|l|}{$\mathrm{CHOP}$} \\
\hline Forward Primer & GGAAACAGAGTGGTCATTCCC \\
\hline Reverse Primer & CTGCTTGAGCCGTTCATTCTC \\
\hline \multicolumn{2}{|l|}{ EIF2AK3 (PERK) } \\
\hline Forward Primer & GGAAACGAGAGCCGGATTTATT \\
\hline Reverse Primer & ACTATGTCCATTATGGCAGCTTC \\
\hline \multicolumn{2}{|l|}{ ERN1 } \\
\hline Forward Primer & CACAGTGACGCTTCCTGAAAC \\
\hline Reverse Primer & GCCATCATTAGGATCTGGGAGA \\
\hline \multicolumn{2}{|l|}{ GRP94 } \\
\hline Forward Primer & GCTGACGATGAAGTTGATGTGG \\
\hline Reverse Primer & CATCCGTCCTTGATCCTTCTCTA \\
\hline \multicolumn{2}{|l|}{ BAX } \\
\hline Forward Primer & CCCGAGAGGTCTTTTTCCGAG \\
\hline Reverse Primer & CCAGCCCATGATGGTTCTGAT \\
\hline BCL2 & \\
\hline
\end{tabular}

Page 12/15 


\begin{tabular}{|ll} 
Forward Primer & GGTGGGGTCATGTGTGTGG \\
\hline Reverse Primer & CGGTTCAGGTACTCAGTCATCC
\end{tabular}

Table 2. Comparison between tonsillar hypertrophy and chronic tonsillitis groups in terms of ER stress and apoptosis pathways.

\begin{tabular}{|c|c|c|c|c|c|c|}
\hline & \multicolumn{2}{|c|}{ Tonsillar hypertrophy $(n=32)$} & \multicolumn{4}{|c|}{ Chronic tonsillitis $(n=32)$} \\
\hline & Mean & SD & Mean & SD & $\mathrm{t}$ & $\mathrm{p}$ \\
\hline \multicolumn{7}{|c|}{ ER stress pathway } \\
\hline ATF4 $\triangle C T$ & 7.01 & 2.18 & 9.41 & 1.11 & -5.540 & $0.000^{\star}$ \\
\hline ATF6 $\triangle C T$ & 2.49 & 1.33 & 6.02 & 1.38 & -10.453 & $0.000 *$ \\
\hline EDEM1 $\triangle C T$ & 3.35 & 2.49 & 4.16 & 1.70 & -1.518 & $0.134^{\star \star}$ \\
\hline CHOP $\triangle C T$ & -2.56 & 1.67 & -0.46 & 3.07 & -3.387 & $0.001^{*}$ \\
\hline$G R P 78 \triangle C T$ & 1.87 & 2.01 & 4.68 & 1.80 & -5.898 & $0.000 *$ \\
\hline EIF2AK3 $\triangle C T$ & -3.03 & 2.19 & -1.23 & 1.77 & -3.610 & $0.000 *$ \\
\hline ERN1 $\triangle C T$ & 1.86 & 2.68 & 4.50 & 2.31 & -4.229 & $0.000^{*}$ \\
\hline$G R P 94 \triangle C T$ & -1.96 & 2.04 & -0.25 & 1.94 & -3.429 & $0.001 *$ \\
\hline \multicolumn{7}{|c|}{ Apoptosis pathway } \\
\hline$B A X \triangle C T$ & 5.04 & 1.47 & 3.12 & 0.90 & 6.302 & $0.000 *$ \\
\hline$B C L-2 \triangle C T$ & 4.60 & 1.84 & 2.76 & 1.96 & -3.879 & $0.000 *$ \\
\hline
\end{tabular}

${ }^{*} p<0.005 ;{ }^{* *} p>0.05$

\section{Figures}




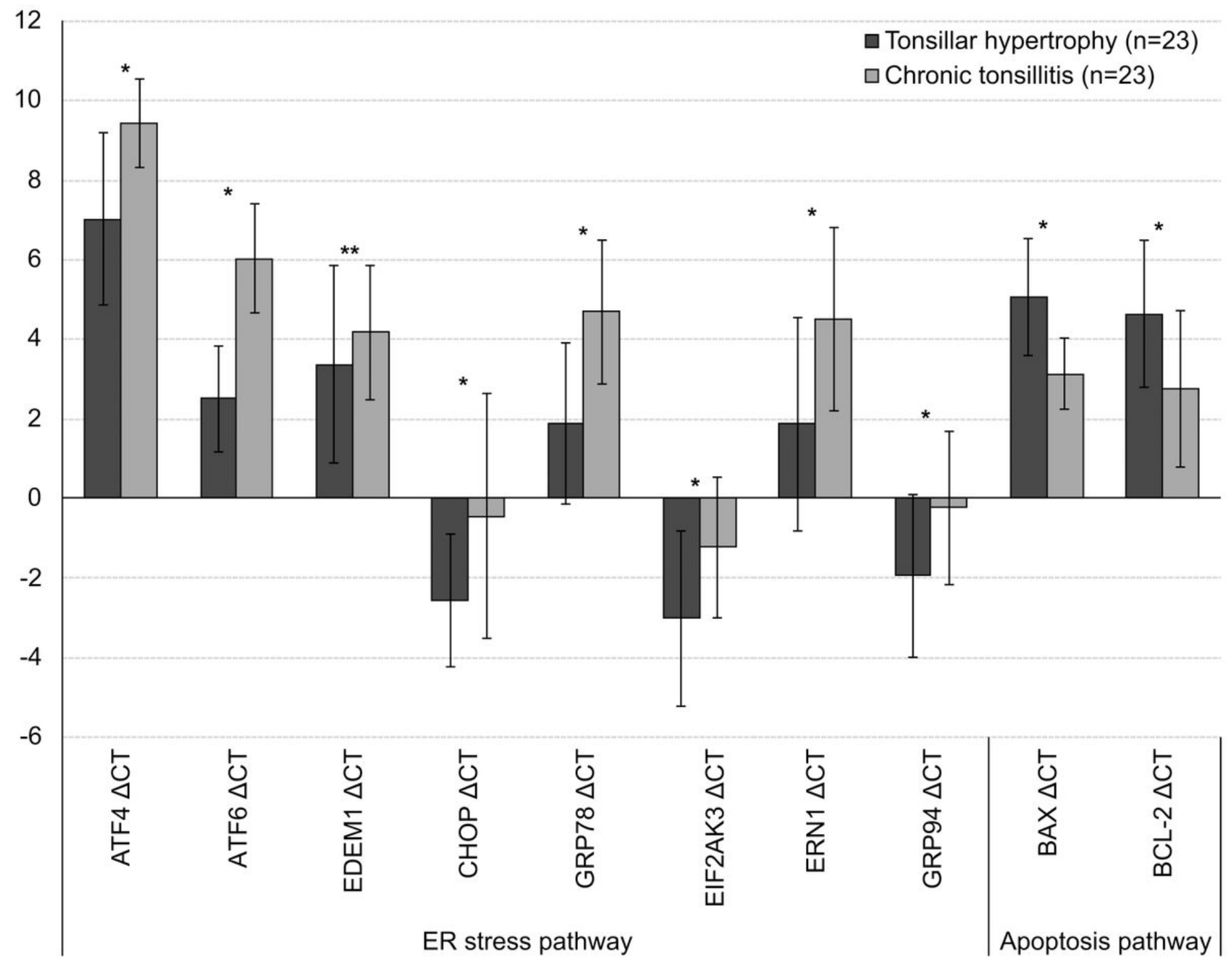

Figure 1

The levels of ER stress and apoptosis markers of the tonsillar hypertrophy group and the chronic tonsillitis group according to the results of Real-time PCR. 

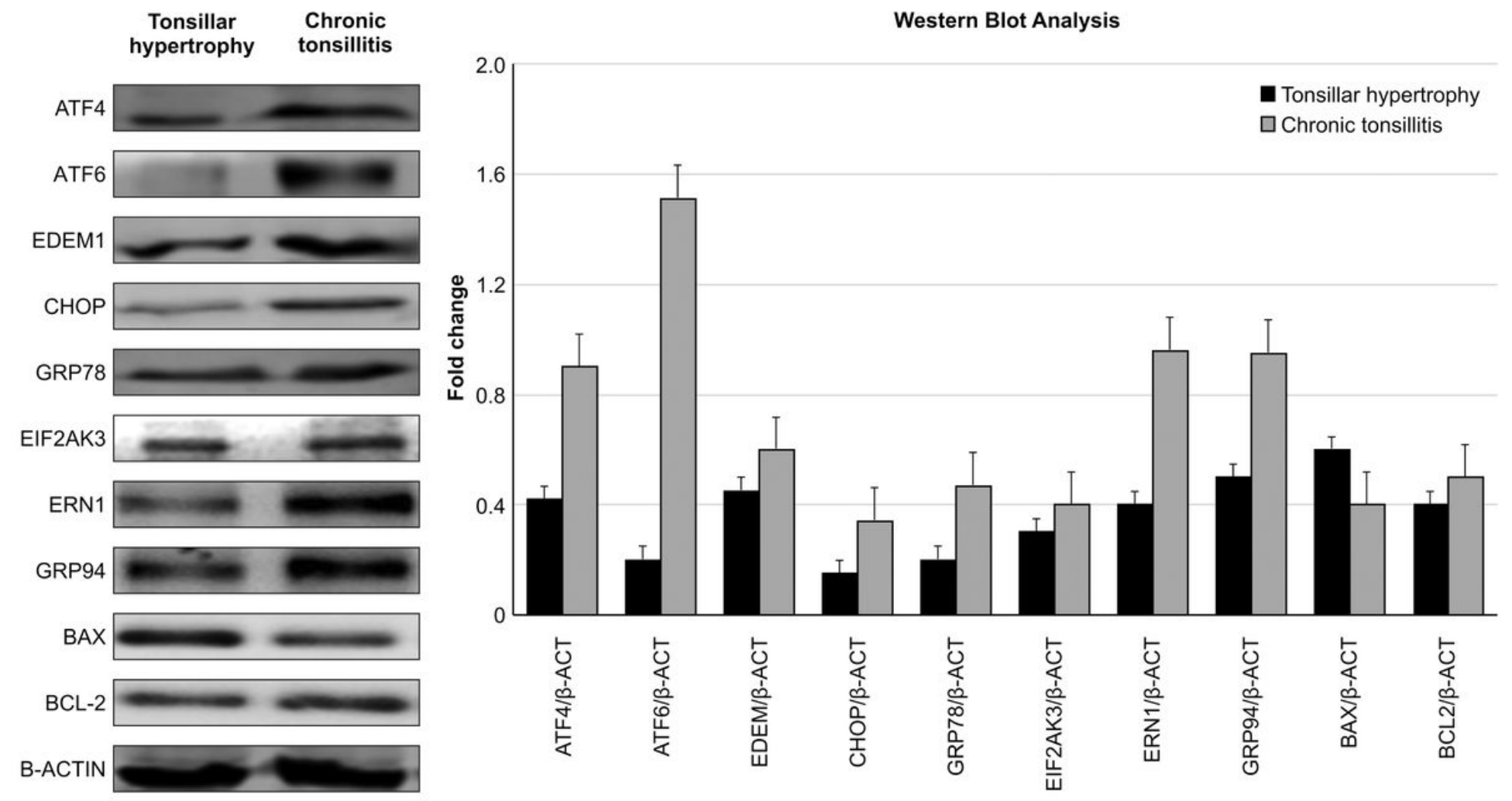

Figure 2

The Western blot analysis of the ER stress and apoptosis markers. 